\title{
Highlights of neuroscience research in Africa
}

\author{
Susan J. van Rensburg • Vivienne A. Russell
}

Received: 4 March 2014 / Accepted: 6 March 2014 / Published online: 19 March 2014

(C) Springer Science+Business Media New York 2014

This special issue highlights recent achievements in neuroscience research in Africa. It reveals some of the diversity of clinical and basic neuroscience research currently being conducted in Africa and opens opportunities for future collaborative research.

The issue begins with an overview of some of the challenges and highlights steps that are being taken to promote neuroscience education and research in Africa. This is followed by reviews of major health concerns in Africa: vertically transmitted HIV, alcohol-induced psychotic disorder, and the increasing problem of prenatal methamphetamine exposure and its devastating effects on cognitive function of the affected children. This is followed by an overview of achievements in basic research in Africa determining the effects of early life stress and exercise on brain function in a rodent model.

Clinical neuroscience research has focused on the major health problems in Africa, as well as risk factors and genetic markers of susceptibility to certain brain disorders. Topics include assessment of cognitive function in patients with HIV, or those who have suffered adversity during the early stages of development, exposure to toxins or drugs of abuse, as well as patients with Addison's disease. Other researchers have investigated the utility of cognitive therapy in bipolar disorder as well as specific tests, including imaging, neuropsychological tests and genotyping, to aid diagnosis of nervous system disorders.

Papers on basic neuroscience focus on elucidating the underlying mechanisms of drugs of addiction, including methamphetamine and khat, as well as neurotoxins such as cyanide, methylmercury and paint thinner. The consequences of glia activation and NMDA receptor inhibition are explored and several papers describe the beneficial effects of honey as well as various drugs and plant extracts in the treatment of different rodent models of human disorders.

S. J. van Rensburg $(\bowtie)$

Division of Chemical Pathology, National Health Laboratory

Service, Tygerberg Hospital, University of Stellenbosch,

P.O. Box 19113, 7505 Tygerberg, South Africa

e-mail: sjvr@sun.ac.za

\section{A. Russell}

Division of Physiology, School of Laboratory Medicine and Medical

Sciences, University of KwaZulu-Natal, 4041 Durban, South Africa

e-mail: vivienne.russell.home@gmail.com 